\title{
Occludin S490 Phosphorylation Regulates Vascular Endothelial Growth Factor-Induced Retinal Neovascularization
}

\author{
Xuwen Liu, ${ }^{*}$ Alyssa Dreffs, ${ }^{*}$ Monica Díaz-Coránguez, ${ }^{*}$ E. Aaron Runkle, ${ }^{\dagger}$ Thomas W. Gardner, ${ }^{*}$ Vince A. Chiodo,
} William W. Hauswirth, ${ }^{\ddagger}$ and David A. Antonetti*

\begin{abstract}
From the Department of Ophthalmology and Visual Sciences, * University of Michigan, Kellogg Eye Center, Ann Arbor, Michigan; the Department of Pathology, ${ }^{\dagger}$ Perelman School of Medicine, University of Pennsylvania, Philadelphia, Pennsylvania; and the Department of Ophthalmology, ${ }^{\ddagger}$ University of Florida, Gainesville, Florida
\end{abstract}

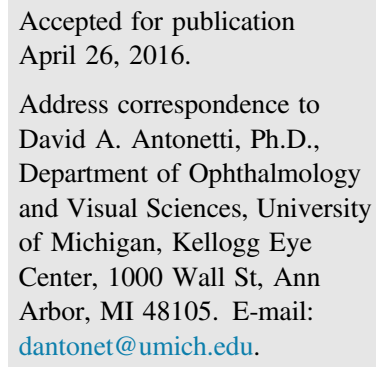

\begin{abstract}
Occludin is a transmembrane tight junction protein that contributes to diverse cellular functions, including control of barrier properties, cell migration, and proliferation. Vascular endothelial growth factor (VEGF) induces phosphorylation of occludin at S490, which is required for VEGF-induced endothelial permeability. Herein, we demonstrate that occludin $\$ 490$ phosphorylation also regulates VEGFinduced retinal endothelial cell proliferation and neovascularization. Using a specific antibody, phospho-occludin was located in centrosomes in endothelial cell cultures, animal models, and human surgical samples of retinal neovessels. 0ccludin $\mathbf{S} 490$ phosphorylation was found to increase with endothelial tube formation in vitro and in vivo during retinal neovascularization after induction of VEGF expression. More important, expression of occludin mutated at $\mathbf{S 4 9 0}$ to Ala, completely inhibited angiogenesis in cell culture models and in vivo. Collectively, these data suggest a novel role for occludin in regulation of endothelial proliferation and angiogenesis in a phosphorylation-dependent manner. These findings may lead to methods of regulating pathological neovascularization by specifically targeting endothelial cell proliferation. (Am J Pathol 2016, 186: 2486-2499; http://dx.doi.org/ 10.1016/j.ajpath.2016.04.018)
\end{abstract}

Retinal neovascularization contributes to severe vision loss in multiple blinding retinal diseases, including retinopathy of prematurity and proliferative diabetic retinopathy (PDR). ${ }^{1-3}$ The neovascular tufts that form are characteristically hyperpermeable and fail to properly perfuse the retina. Vascular endothelial growth factor (VEGF) has been causally linked to both hyperpermeability and pathological retinal angiogenesis, and therapies targeting VEGF effectively reduce both retinal edema and neovascularization in diabetic retinopathy and acute macular degeneration. ${ }^{4-6}$ However, not all patients respond to anti-VEGF therapy and the need for repeat intraocular injections and the associated risk of complications suggest that better understanding the mechanisms of VEGFinduced vascular permeability and angiogenesis may lead to improved therapeutic options for these blinding eye diseases.

Occludin is a member of the tight junction complex in the blood-brain and blood-retinal barriers (BRB), but the function of occludin appears complex and incompletely understood. Occludin gene deletion in mice leads to a host of phenotypic alterations, including growth retardation, loss of the blood-testis barrier, and brain calcification, ${ }^{7}$ and leads to hyperproliferation of mucous epithelial cells in the intestinal lining, ${ }^{8}$ suggesting occludin contributes a role in cell growth and differentiation. Indeed, oncogenic transformation of a variety of cell types is associated with altered occludin expression. ${ }^{9}$ Furthermore, reduction of occludin content using

Supported by NIH grant EY012021 (D.A.A.), the Jules and Doris Stein Professorship from Research to Prevent Blindness (D.A.A.), the Taubman Institute (T.W.G.), and core NIH grants EY007003 (Core Center for Vision Research at the Kellogg Eye Center) and DK020572 (Morphology and Image Analysis Core of the Michigan Diabetes Research and Training Center).

Disclosures: None declared. 
siRNA in ARPE-19 cells, a human retinal pigmented epithelial cell line, increases DNA synthetic rate and cell proliferation. ${ }^{10}$ Conversely, expression of exogenous occludin suppresses tumor growth in nude mice of Raf1-transformed rat salivary gland epithelial cells. ${ }^{11}$ Finally, occludin targets transforming growth factor- $\beta$ receptor to the junctional complex, promoting efficient epithelial to mesenchymal transition. ${ }^{12}$ Collectively, these studies provide strong evidence for a role of occludin in proliferation, but the contribution to endothelial angiogenesis has not been investigated.

Studies from numerous laboratories demonstrate that occludin phosphorylation contributes to binding interactions with the junction organizing protein, zonula occludens 1 (ZO-1) and regulation of barrier properties. ${ }^{13}$ We previously identified occludin S490 as a phosphorylation site on the second turn of the terminal coiled-coil domain of occludin that is required for VEGF-induced occludin ubiquitination and intracellular trafficking that regulates vascular permeability ${ }^{14,15}$ in a protein kinase C $\beta$ dependent manner. ${ }^{16}$ Further studies revealed a novel function of occludin as a regulator of centrosome separation and mitotic entry in MDCK cells, and expression of occludin mutated at S490 to Ala slowed cell proliferation and hindered mitotic entry because of delayed centrosome separation. ${ }^{17}$

Herein, we show that occludin S490 phosphorylation is required for VEGF-induced neovascularization in cell culture and in vivo. Occludin phosphorylated on S490 was detected in centrosomes of proliferating endothelial cells, in vivo in mouse retinal whole mounts with proliferating vessels, and in human surgical samples of retinal neovascularization. Occludin S490 phosphorylation increases as endothelial cells are induced to form tubes in the presence of VEGF and expression of S490A occludin completely inhibits endothelial cell proliferation, migration, and tube formation induced by VEGF. An siRNA knockdown of occludin promoted tube formation both basally and after VEGF induction in retinal endothelial cells, suggesting occludin acts to inhibit angiogenesis that may be relieved by occludin knockdown or S490 phosphorylation. Furthermore, increased occludin S490 phosphorylation is associated with pathological retinal angiogenesis in mice, as observed using doxycyclineinducible VEGF expression from photoreceptors and subsequent neovascularization. More important, viral delivery of S490A occludin completely blocked VEGF-induced retinal neovascularization. These studies demonstrate that occludin S490 phosphorylation is required for VEGFinduced retinal neovascularization and implicate a critical role for occludin in angiogenesis.

\section{Materials and Methods}

\section{Reagents}

Recombinant human VEGF165 was from R\&D Systems (Minneapolis, MN). Complete protease inhibitor cocktail tablets and PhosSTOP phosphatase inhibitor cocktail tablets were from Roche (Indianapolis, IN). Type 1 collagenase was from Worthington Biochemical (Lakewood, NJ). SYTO 13 green fluorescent nucleic acid stain was from Life Technologies (Carlsbad, CA). Bovine retinal endothelial cells (BRECs) were transfected with siRNA (5'-AUAAAGAACUGGAUGACUAUU-3', siOcc1; or 5'-GUAAACAGUUGAAGAGCAAUU-3', siOcc2) or a nonspecific control siRNA (Dharmacon, Lafayette, CO). All other chemical reagents were from EMD Millipore (Billerica, MA) or Sigma-Aldrich (St. Louis, MO).

\section{Cell Culture and Nocodazole Synchronization}

BRECs were isolated and cultured as described previously. ${ }^{15}$ BRECs grown on two-dimensional bovine type I collagen film were arrested in prometaphase with $200 \mathrm{ng} / \mathrm{mL}$ nocodazole for 16 hours, followed by release into fresh media and harvesting at the indicated time points for Western blot analysis. Bovine type I collagen solution (Advanced BioMatrix, San Diego, CA) was used to prepare thin films of two-dimensional culture or hydrogels for three-dimensional (3D) culture by a modification of protocol described previously. ${ }^{18,19}$ Briefly, a stock solution of collagen at $3 \mathrm{mg} / \mathrm{mL}$ was diluted and mixed in a 4:1 ratio with $5 \times$ MCDB-131 media containing HEPES (20 $\mathrm{mmol} / \mathrm{L}), \mathrm{NaHCO}_{3}(24 \mathrm{mmol} / \mathrm{L})$, fibronectin $(2.5 \mu \mathrm{g} / \mathrm{mL})$, and laminin $(2.5 \mu \mathrm{g} / \mathrm{mL})$. The mixed collagen solution was neutralized with $\mathrm{NaOH}(0.01 \mathrm{~N})$ and kept at $4^{\circ} \mathrm{C}$ to minimize polymerization. To obtain a thin film, collagen mixture $(0.125$ $\mathrm{mL} / \mathrm{cm}^{2}$ ) was coated then immediately removed for each well and kept at $37^{\circ} \mathrm{C}$ for 2 hours to allow complete gelling.

\section{D Collagen Tube Formation Assay}

For the lower gel layer, $400 \mu \mathrm{L}$ of the collagen gel mixture was added to each well of 24-well plates and incubated at $37^{\circ} \mathrm{C}$ for 2 hours. After polymerization, $7.5 \times 10^{4}$ BRECs were seeded in each well and cultured for 24 hours. The medium was then removed and $150 \mu \mathrm{L}$ of the collagen gel mixture was added to each well. The plates were incubated at $37^{\circ} \mathrm{C}$ for 2 hours to allow polymerization of the upper gel layer. Last, $500 \mu \mathrm{L}$ of $1 \%$ fetal bovine serum step down medium with vehicle or VEGF was added. Tube growth was monitored for up to 24 hours or terminated as described later. Calcein-AM $(2 \mu \mathrm{g} / \mathrm{mL}$; Life Technologies) was added directly to the culture medium 30 minutes before fixing and imaging tubes at $\times 10$ magnification. Three different fields per well were randomly chosen and photographed using a model Eclipse TE300 inverted phase microscope (Nikon, Tokyo, Japan). The number of tubes that were $>100 \mu \mathrm{m}$ was counted using MetaMorph software version 7.6.3 (Molecular Devices, Sunnyvale, CA).

\section{Western Blot Analysis}

Cells were lysed in detergent-based buffer as described previously. ${ }^{20}$ BRECs grown in collagen gel were lysed after dissolving the collagen gels with $1 \mathrm{mg} / \mathrm{mL}$ type I collagenase. 
Mice retinas were harvested and quickly frozen in liquid nitrogen, and subsequently lysed with the same buffer. After centrifugation, supernatants were applied to NuPAGE SDSPAGE gels (Life Technologies, Carlsbad, CA) followed by transfer to nitrocellulose membrane. Membranes were blocked in 2\% ECL Prime Blocking Reagent diluted in $1 \times$ tris-buffered saline and Tween 20. Membranes were then incubated with rabbit anti-occludin phospho-S490 (1:250) ${ }^{14}$ mouse anti-occludin and mouse anti-claudin-5 (1:1000; Life Technologies, Carlsbad, CA), rabbit anti-histone H3 phospho-S-10 (1:1000; Cell Signaling, Danvers, MA), mouse anti-actin (1:5000; Cell Signaling), phospho-VEGF receptor 2 (Tyr1175) (D5B11), rabbit mAb and VEGF receptor 2 (55B11) rabbit mAb (1:1000; Cell Signaling), mouse anti-phospho-p44/42 MAPK (ERK1/2), and rabbit anti-p44/42 MAPK (1:1000; Cell Signaling). Primary antibodies were detected using goat anti-mouse or anti-rabbit secondary antibody conjugated with horseradish peroxidase $(1: 10,000)$ or goat anti-mouse or anti-rabbit secondary antibody conjugated with alkaline phosphatase $(1: 10,000)$ and chemiluminescence with horseradish peroxidase substrate Lumigen TMA-6 (Lumigen, Southfield, MI) or AP substrate ECF (GE Healthcare, Piscataway, NJ).

\section{Immunostaining}

Immunohistochemistry was performed as described previously. ${ }^{21}$ Briefly, cells were incubated with primary antibodies mouse anti- $\gamma$-tubulin (1:200; Abcam, Cambridge, MA), mouse/rabbit anti-occludin (1:200; Life Technologies), rabbit anti-occludin phospho-S490 (1:250) for 2 days at $4^{\circ} \mathrm{C}$, washed, and then incubated with secondary antibodies goat anti-mouse Alexa Fluor 488 (1:400; Life Technologies), goat anti-rabbit Alexa Fluor 555 (1:400; Life Technologies), and Hoechst (1:1000; Life Technologies) overnight at $4^{\circ} \mathrm{C}$. Mice retinal flat mounts or cryostat sections were blocked in $10 \%$ donkey serum with $0.3 \%$ Triton X-100. Samples were then incubated with rabbit anti-occludin (N-Term) (1:200; Life Technologies), rabbit anti-ZO-1 (Life Technologies, 1:50), rat anti-Ki-67 (1:100; eBiosciences, San Diego, CA) antibodies, and isolectin GS-IB4 Alexa Fluor 647 (1:50; Life Technologies) for 3 days, followed with Rhodamine Red-Xconjugated donkey anti-rabbit IgG (1:200; Jackson ImmunoResearch Laboratories, West Grove, PA), Alexa Fluor 488-conjugated donkey anti-rat (1:400; Jackson ImmunoResearch Laboratories) secondary fluorescent antibodies. Cells and retina samples were imaged using a confocal microscope (TCS SP5; Leica, Wetzlar, Germany).

For colocalization analysis, the confocal microscope images were analyzed using Imaris software version 7.3 (Bitplane USA, Concord, MA) to determine Pearson's coefficient of colocalization.

\section{Cell and Biochemical Assays}

Occludin mutants were described previously. ${ }^{15}$ In brief, S490 of human occludin (Occ) in pENTR221 (Invitrogen,
Rockville, MD) was substituted to alanine (S490A) or aspartic acid (S490D) and cDNA of these mutants or wildtype human occludin (WT-Occ) were transferred into pmaxFP-Green-C expression vector (Lonza, Walkersville, MD). All constructs were confirmed by sequencing. Transfections with plasmids containing occludin or its mutants were performed using Amaxa Nucleofector System (Amaxa, Koeln, Germany). BREC proliferation assay was performed according to a modified protocol of the Click-iT EdU Alexa Fluor 594 Imaging kit (Life Technologies). BREC migration assay was performed using modified Boyden chambers (8.0- $\mu \mathrm{m}$ PET Membrane 24-well Cell Culture Inserts; BD Biosciences, San Jose, CA) with modification from the previously described method. ${ }^{22}$ Briefly, BRECs transfected with occludin or mutants were resuspended in $1 \%$ fetal bovine serum step down medium and seeded at $5 \times 10^{3}$ cells per well in Transwell upper chambers with both top and bottom of the membranes coated with $50 \mu \mathrm{g} / \mathrm{mL}$ type I collagen. Cells were induced to migrate by addition of $50 \mathrm{ng} / \mathrm{mL}$ VEGF, placed in the bottom chamber, for 6 hours. Cells that migrated to the bottom of the chamber were counted after SYTO13 Green Fluorescent Nucleic Acid staining. Three different fields per well were randomly chosen and photographed using a model Eclipse TE300 inverted phase microscope (Nikon, Tokyo, Japan). The values are expressed as number of migrated cells per $\times 10$ power field.

\section{Animals}

All animal experiments were conducted in accordance with the Association for Research in Vision and Ophthalmology Statement for the Use of Animals in Ophthalmic and Vision Research and the guidelines of the University Committee on Use and Care of Animals at the University of Michigan. Double-transgenic rho/rtTA-TRE/VEGF mice with doxycycline-inducible expression of VEGF in retina were obtained from Dr. Peter Campochiaro (Johns Hopkins Wilmer Eye Institute, Baltimore, MD). ${ }^{23}$ Mice, aged 6 to 8 weeks old, were given an initial oral gavage then provided drinking water containing $2 \mathrm{mg} / \mathrm{mL}$ of doxycycline or pure water for the control group. Doxycycline-treated and control mice were sacrificed at various time points after gavage feeding as indicated later. A recombinant AAV serotype 2 quadruple tyrosine to phenylalanine (Y-F) capsid mutant containing the vascular endothelial cadherin promoter was used to drive human occludin cDNA or Occludin S490A mutant expression. AAV vectors were packaged and purified as previously described. ${ }^{24}$ Subretinal injections were performed as previously described ${ }^{25,26}$ using a tapered pulled glass pipette inserted into a sclerotomy and connected to a nanoinjector (Nanoinjector II; Drummond Scientific Company, Broomall, PA), which allowed for a slow injection of 1 $\mu \mathrm{L}$ of virus at $2.0 \times 10^{13}$ genome copies per mL. The subretinal injection was performed with direct visualization through a dissecting microscope. Only animals with minimal surgical complications were retained for further evaluation. 
For measures of BRB integrity using Sulfo-NHS-Biotin deposition, mice were deeply anesthetized and perfused with $10 \mathrm{~mL}$ of Sulfo-NHS-LC-Biotin (Thermo Scientific) at $0.5 \mathrm{mg} / \mathrm{mL}$ in phosphate-buffered saline by transcardiac perfusion, followed by flushing with $1 \%$ paraformaldehyde (PFA) in phosphate-buffered saline. Eyes were postfixed in $1 \%$ PFA at $4^{\circ} \mathrm{C}$ for 6 hours before retina dissection. BRB permeability measured by fluorescein isothiocyanatebovine serum albumin accumulation was described previously. ${ }^{27}$ Briefly, fluorescein isothiocyanate-bovine serum albumin $(100 \mathrm{mg} / \mathrm{kg}$ body weight) was injected in femoral vein and allowed to circulate for 10 minutes. Eyes were enucleated and fixed in 4\% PFA. Retinas were dissected and subjected to cryosectioning and subsequent imaging analysis. Whole mount retinas were incubated with rat anti-Ki-67 (eBiosciences) antibody and Isolectin GS-IB4 Alexa Fluor 647 (Life Technologies) in 10\% donkey serum with $0.3 \%$ Triton X-100 for 3 days, followed with Texas Red Streptavidin (Vector) and Alex Fluor 488-conjugated donkey anti-rat (1:400; Jackson ImmunoResearch) secondary fluorescent antibody. Retina samples were imaged using a confocal microscope (TCS SP5; Leica, Wetzlar, Germany). To quantify the loss of tight junction proteins in immunofluorescence images, a confocal Z-stack of 10 images were collected over a depth of $5 \mu \mathrm{m}$ and projected as a single image. For each condition, images of four random fields from three independent experiments were taken. Occludin and ZO-1 border staining were quantified by a rank scoring system as described previously. ${ }^{28}$ Scoring was completed in a masked manner by three impartial observers who were provided scoring standard images for comparison. For each condition, images of four random fields from six retinas, or 24 images total, were taken and the frequency of each ranking score was determined.

\section{Epiretinal Membranes of Human Proliferative Diabetic Retinopathy}

Epiretinal fibrovascular membranes were obtained from six patients with active PDR during pars plana vitrectomy for the removal of vitreous hemorrhage and/or the repair of tractional retinal detachment. The Institutional Review Board of the University of Michigan approved the protocol. The severity of retinal neovascular activity was graded clinically at the time of vitrectomy using previously published criteria. ${ }^{29}$ Partly active PDR was present in all six patients used for the current study. Membranes were fixed in 4\% PFA for 15 minutes, and $5 \mu \mathrm{m}$ cryostat sections were prepared for immunostaining.

\section{Statistical Analysis}

All experiments were repeated three or more times and expressed as means \pm SEM and were analyzed using twotailed Student's $t$-test (two conditions) or analysis of variance (three or more conditions) using Prism software version 5.0 (GraphPad Software, La Jolla, CA). $P<0.05$ was considered statistically significant.

\section{Results}

Occludin Is Phosphorylated on S490 during Mitosis and Colocalizes with Centrosomes in Proliferating Endothelial Cells and Human Retinal Neovascular Tufts

Occludin is typically identified at cell borders in the tight junction complex in confluent cell monolayers. However, immunofluorescence analysis of proliferating primary BRECs reveals phospho-occludin localization in centrosomes. Mitotic cells were identified on the basis of nuclear condensation patterns observed by Hoechst staining using confocal microscopy. Immunofluorescence staining using a phosphosite-specific antibody demonstrated that occludin pS490 colocalized with the centrosome marker $\gamma$-tubulin throughout mitosis (Figure 1, A and B) as well as cytoplasmic staining. Quantification of colocalization of $\mathrm{pS} 490$ and $\gamma$-tubulin in confocal images using Imaris 7 software reveals a Pearson coefficient of colocalization of 0.61 in mitotic cells compared to 0.07 in nonmitotic cells. Centrosomal location of occludin was confirmed in BRECs transfected with green fluorescent protein (GFP)-tagged WT occludin and occludin S490D (Figure 1C). These data demonstrate that occludin phosphorylated at S490 is present at mitotic centrosomes of BRECs.

This same occludin S490 phosphorylation was observed in human retinal neovascular tufts of epiretinal membranes. Epiretinal fibrovascular membranes from six patients with active PDR were immunostained with Ki-67 antibody to identify proliferative cells, $\gamma$-tubulin antibody, and occludin pS490-specific antibody. Figure 1D shows a fundus image of a PDR patient and Figure 1E shows hematoxylin and eosin-stained surgically removed membranes. Immunofluorescence microscopy of the membranes reveals clear colocalization of occludin pS490 with $\gamma$-tubulin in centrosomes of actively proliferating vascular cells (Figure $1 \mathrm{~F}$ ). In fact, occludin pS490 costaining with the centrosomal marker could be observed in all six active PDR surgical samples. These studies demonstrate occludin phosphorylation at the centrosome in actively proliferating endothelial cells, including human retinal neovascularization.

\section{Occludin S490 Is Phosphorylated during VEGF-Induced BREC Tube Formation}

To test the hypothesis that phosphorylation of occludin S490 contributes to the regulation vascular angiogenesis, occludin S490 phosphorylation was monitored during VEGF-induced BREC tube formation. Primary BRECs were subjected to a classic tube formation assay in $3 \mathrm{D}$ collagen matrix in the presence or absence of VEGF. Clear evidence of tube formation was observed during a 24-hour time course, with tube growth occurring between 12 and 24 
A

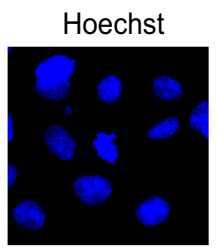

Y-tubulin
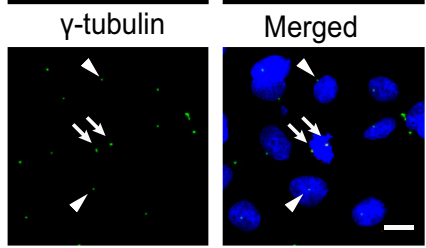

C

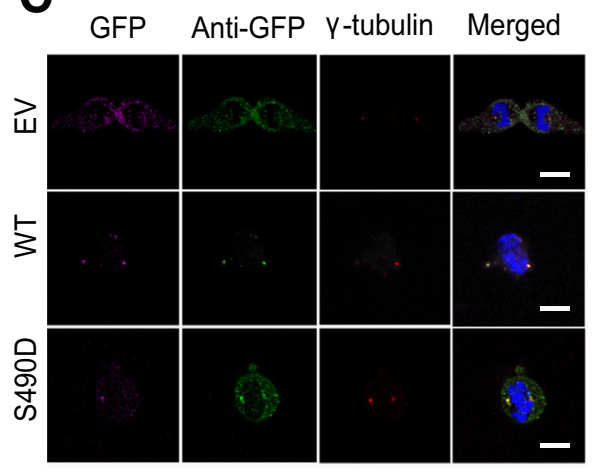

$\mathbf{E}$

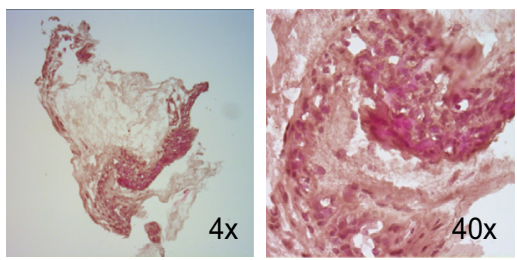

B

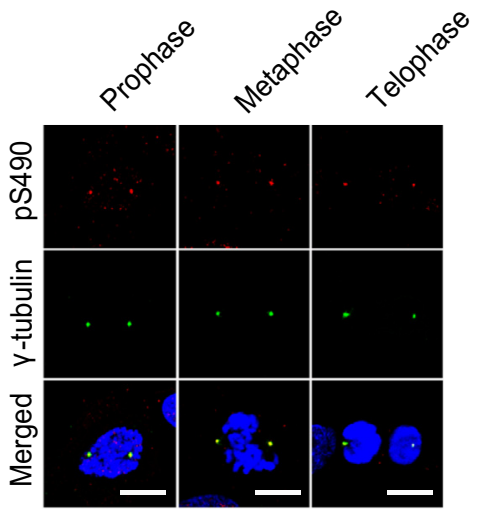

D

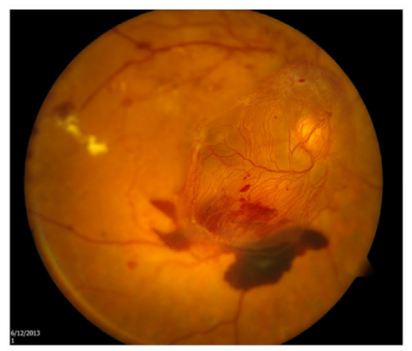

$\mathbf{F}$

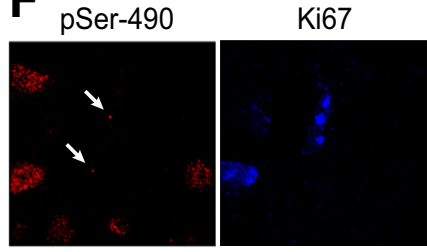

pSer-490+y-tubulin Merged

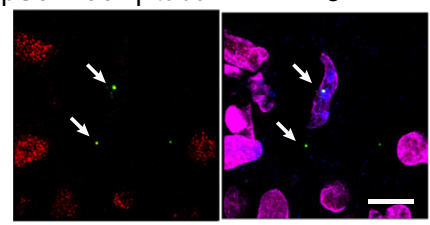

Figure 1 Occludin S490 phosphorylation in centrosome. A and B: Bovine retinal endothelial cells (BRECs) were fixed and stained with pS490specific antibody (red), the centrosome marker $\gamma$-tubulin (green), or DNA using Hoechst dye (blue). The arrows indicate the centrosomes of the proliferating cell, and the arrowheads indicate the centrosomes of the nonproliferating cell. Pearson coefficient of colocalization of pS490 and $\gamma$-tubulin was 0.61 (mitotic cells, $n=60$ ) and 0.07 (nonmitotic cells, $n=60$ ). C: BRECs transfected with empty vector (EV), wild-type (WT) Occ, or S490D 0cc were fixed and observed for green fluorescent protein (GFP; faux color in purple), stained with antibodies for GFP (green), $\gamma$-tubulin (red), and DNA (Hoechst dye, blue). D: Fundus image of patient with proliferative diabetic retinopathy and hemorrhage. E: Epiretinal membrane stained with hematoxylin and eosin. F: Immunohistochemistry for occludin pS490 (red), $\gamma$-tubulin (green), Ki-67 (blue), and DAPI (magenta) in human epiretinal membrane. The arrows indicate that occludin $\$ 490$ phosphorylation can be observed in the centrosomes of proliferating endothelial cells in the active proliferative diabetic retinopathy membrane. Scale bar $=10 \mu \mathrm{m}$ $(\mathbf{A}-\mathbf{C}, \mathbf{F})$. hours (Figure 2A). Immunoblotting cell lysates for pS490 revealed two clear bands, one at just over $50 \mathrm{kDa}$ (the expected molecular weight of occludin) and a prominent band at approximately $66 \mathrm{kDa}$ previously shown by peptide blocking and immunoprecipitation experiments to represent S490 phosphorylated occludin (Figure 2B). ${ }^{14}$ Quantification revealed that the amount of $\mathrm{pS} 490$ occludin $(66 \mathrm{kDa})$ relative to total occludin significantly increased by 12 and 24 hours as tube formation proceeds (Figure 2C).

Phosphorylation of occludin at S490 was also observed in synchronized endothelial cell proliferation. Proliferating endothelial cells on two-dimensional matrices were inhibited at prometaphase with nocodazole and then the drug was washed out to release the cells from the mitotic cell cycle block. Asynchronous cells showed low levels of occludin S490 phosphorylation, but the phosphorylation of occludin dramatically increased with nocodazole block and subsequent release (Figure 2D). This increase in occludin phosphorylation was statistically correlated with an increase in the mitotic phase marker, phosphorylation of histone H3 on S10, by Spearman's rank correlation (Figure 2, E and F). Spearman's rank correlation coefficient between pS490 occludin and histone $\mathrm{H} 3(\mathrm{pS} 10)$ is $0.9429, P<0.05$. 
A
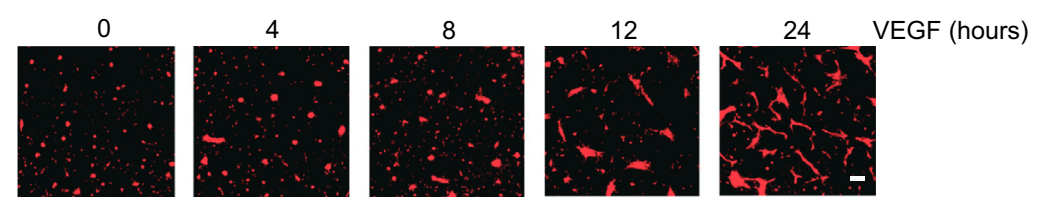

\section{B}

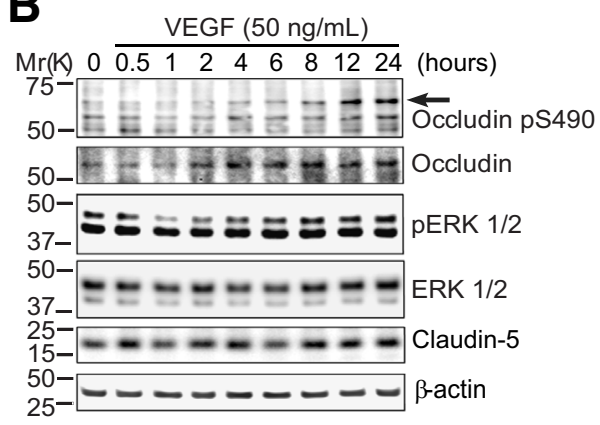

D

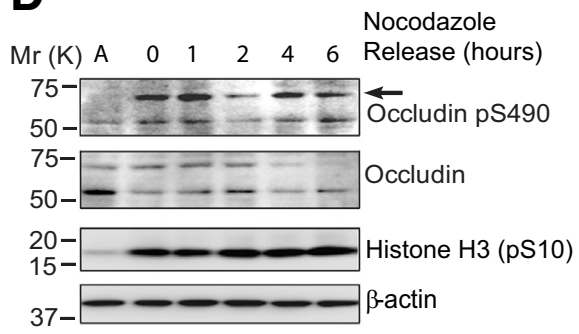

C

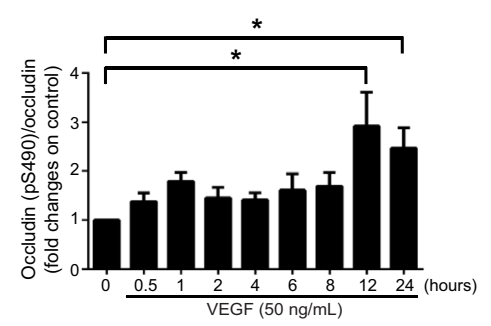

E

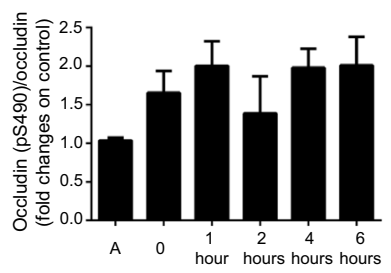

$\mathbf{F}$

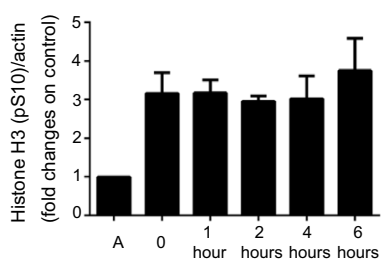

Figure 2 Occludin $\mathbf{S 4 9 0}$ is phosphorylated during vascular endothelial growth factor (VEGF) induced tube formation. A: Bovine retinal endothelial cells (BRECS) grown in three-dimensional collagen gels were treated with VEGF and stained with Calcein AM. B: BREC tubes were lysed fo Western blot analysis after dissolving the gels with collagenase. C: Quantification of occludin pS490 from three independent experiments. D: BREC monolayers were arrested in prometaphase using nocodazole followed by release and harvesting at the indicated time points for Western blot. Quantification of the content of occludin pS490 (E) and histone $\mathrm{H} 3$ (pS10) (F) from three independent experiments. Spearman's rank correlation coefficient between pS490 Occ and histone $\mathrm{H} 3$ $(\mathrm{pS} 10)$ is $0.9429, P<0.05$. Data represent means \pm SEM with analysis by one-way analysis of variance with Bonferroni post-test $(\mathbf{C}) .{ }^{\star} P<0.05$. Scale bar $=100 \mu \mathrm{m}(\mathbf{A})$. A, asynchronous cells; $\mathrm{Mr}$ $(\mathrm{K})$, relative molecular mass (kilodalton).
Inhibition of Occludin S490 Phosphorylation Reduces Endothelial Cell Proliferation, Migration, and Tube Formation

To determine whether occludin S490 phosphorylation is required for angiogenesis, BRECs were transfected with occludin S490A mutant and compared to cells with Wt Occ or empty vector expressing GFP only, using wellestablished models of angiogenesis, including proliferation, migration, and tube formation. Measures of endothelial proliferation in response to VEGF were made by either ${ }^{3} \mathrm{H}$ thymidine incorporation on two-dimensional culture (Supplemental Figure S1) or Click-iT EdU incorporation in 3D culture (Figure 3A). This analysis revealed that transfection of occludin S490A mutant inhibited VEGF-induced proliferation as measured by DNA synthesis. Migration of endothelial cells across a Transwell porous filter toward VEGF was also inhibited with occludin S490A mutant (Figure 3B). More important, tube formation assays demonstrated that S490A mutant completely prevented VEGF-induced tube formation, whereas no effect was observed with the S490D mutant (Figure 3, C and D).
Previous research identified S471 as another phosphorylation site on the first turn of the occludin coiled-coil domain. ${ }^{14}$ Transfection of S471A and S471D mutants had no effect on tube formation demonstrating specificity of the S490 site toward inhibition of VEGF-induced angiogenesis in BRECs. Collectively, these studies provide compelling evidence that occludin phosphorylation at S490 is required for VEGF-induced endothelial angiogenesis in cell culture assays and that expression of the non-phosphorylatable occludin S490A point mutant acts in a dominant negative manner.

To determine whether S490A Occ expression inhibited VEGFR2 receptor tyrosine kinase signaling, we examined VEGFR signaling in endothelial cells transfected with GFP, $\mathrm{Wt}$, or S490A Occ. Western blotting confirmed that Wt Occ or S490A Occ was successfully transfected in BRECs (Supplemental Figure S2). Transfection of S490A point mutant did not inhibit either VEGFR2 receptor phosphorylation as measured by Tyr1175 phospho-specific antibody blotting or downstream ERK1/2 phosphorylation in cells treated with VEGF for 15 minutes (Supplemental Figure S2). These results demonstrate that expression of 
A

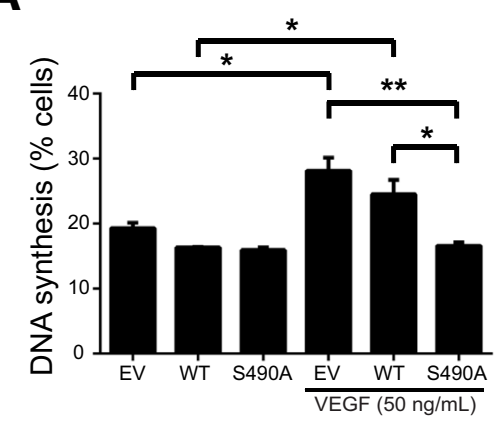

B

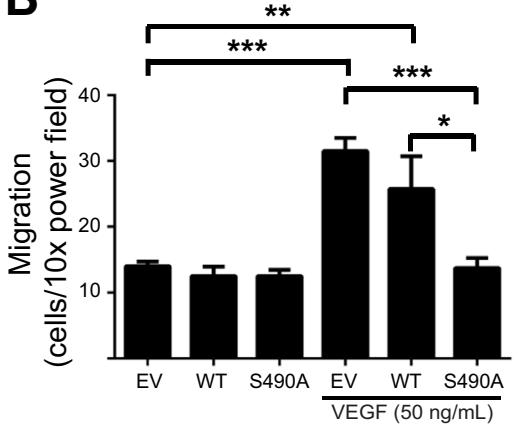

C
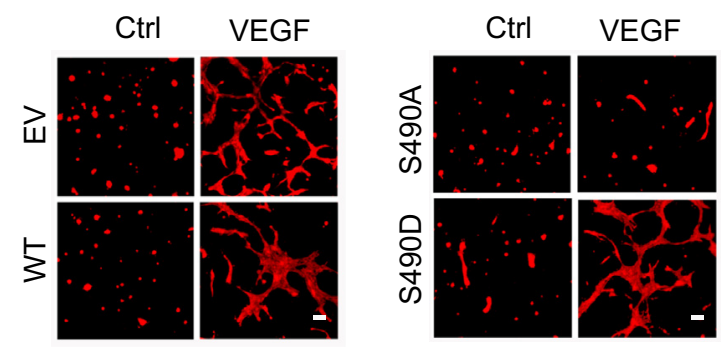

D

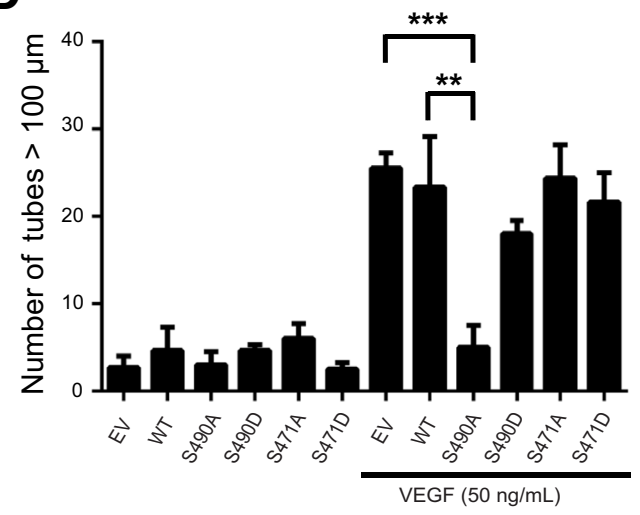

occludin S490A does not block angiogenesis by inhibiting receptor tyrosine kinase activation.

\section{Occludin Knockdown Promotes Endothelial Cell Proliferation and Tube Formation}

To determine the contribution of occludin to control of VEGF-induced endothelial cell growth and tube formation, occludin expression was reduced by transfecting siRNA targeting occludin in BREC. Tube formation assays demonstrated that the occludin knockdown by either SiOcc1 or SiOcc2 significantly promoted endothelial tube formation, both basally and after VEGF induction in BRECs (Figure 4, A and B; Supplemental Figure S3, A and B). Add back experiments revealed that cotransfection of both WT and S490A mutant occludin prevented VEGF-induced tube formation, whereas the S490D mutant failed to reverse the effect of occludin knockdown on tube formation (Figure 4E). Furthermore, Click-iT EdU DNA synthesis assay in 3D
Figure 3 S490A occludin prevents vascular endothelial growth factor (VEGF)-induced endothelial cell proliferation, migration, and tube formation. A: Bovine retinal endothelial cells (BRECS) were transfected with empty vector (EV), wild-type (WT) $0 \mathrm{cc}$, and S490A 0cc and treated with VEGF and DNA synthesis was measured after 8 hours (Click-iT EdU), quantification from three independent experiments. B: Transfected BRECs were plated on Transwells and induced to migrate toward VEGF for 6 hours and stained with SYTO 13 green. Quantification of three independent experiments performed with triplicate wells. C: Transfected BRECS were grown in three-dimensional bovine type I collagen gel for 24 hours followed by treatment with VEGF. At 24 hours, cells were stained with Calcein AM before fixing and imaging. D: The number of tubes $>100 \mu \mathrm{m}$ was quantified in three independent experiments. Data represent means \pm SEM with analysis by one-way analysis of variance with Bonferroni post-test (A, B, and D). ${ }^{\star} P<0.05$, ${ }^{* *} P<0.01$, and ${ }^{* * *} P<0.001$. Scale bar $=100$ $\mu \mathrm{m}$ (C). Ctrl, control. culture revealed that occludin knockdown promoted BREC proliferation by approximately $100 \%$ in the absence of VEGF (Figure 4, C and D, and Supplemental Figure S3, C and D) as compared with scramble siRNA transfected BREC. These data are in good agreement with previously published data revealing a similar knockdown of occludin leading to increased ARPE19 epithelial cell proliferation. ${ }^{10}$ Collectively, the data suggest that occludin inhibits endothelial proliferation and either reduction of occludin content or S490 phosphorylation may relieve this inhibition.

\section{Occludin S490 Is Phosphorylated during VEGF-Induced Retinal Angiogenesis}

Increased S490 phosphorylation was also observed in mouse retina in vivo in a model of retinal neovascularization. The tetracycline-inducible expression of VEGF from rods was achieved using previously described mice ${ }^{23}$ with rhodopsindriven expression of reverse tetracycline transactivator 
A

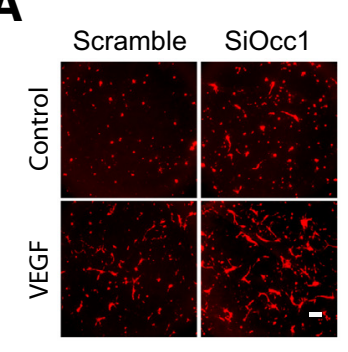

B

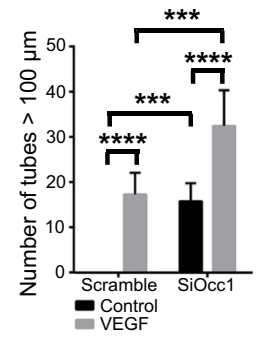

C

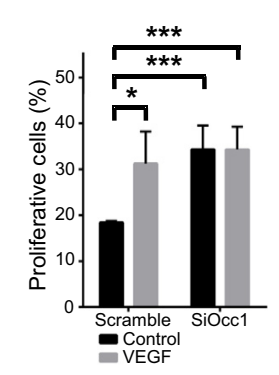

D

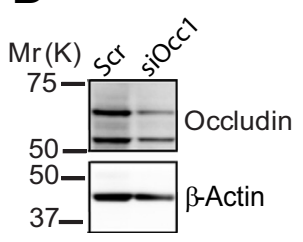

$\mathbf{E}$

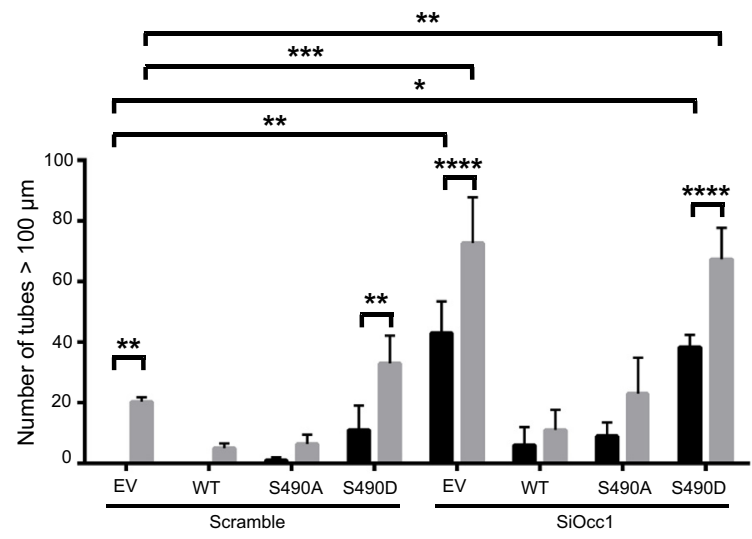

Figure 4 Knockdown of occludin increases bovine retinal endothelial cell (BREC) tube formation and proliferation. A: BRECs were transfected with $100 \mathrm{nmol} / \mathrm{L}$ scramble siRNA or $100 \mathrm{nmol} / \mathrm{L}$ siOcc1 and subjected to tube formation assays. B: Tubes $>100 \mu \mathrm{m}$ were quantified from three independent experiments. C: DNA synthesis with quantification of three independent experiments. Data shown represent analysis by Student's t-test. D: Western blot analysis. E: BRECs were cotransfected with 100 $\mathrm{nmol} / \mathrm{L}$ scramble siRNA or $100 \mathrm{nmol} / \mathrm{L}$ si0cc1 and empty vector (EV), wildtype (WT) Occ, S490A 0cc, or S490D 0cc and subjected to tube formation assays. Tubes $>100 \mu \mathrm{m}$ were quantified from three independent experiments. Data shown in $\mathbf{B}$ and $\mathbf{E}$ represent analysis by two-way analysis of variance with Bonferroni post-test. Data represent means \pm SEM (B, C, and E). ${ }^{*} P<0.05,{ }^{* *} P<0.01,{ }^{* * *} P<0.001$, and ${ }^{* * * *} P<0.0001$. Scale bar $=100 \mu \mathrm{m}(\mathbf{A}) . \operatorname{Mr}(\mathrm{K})$, relative molecular mass (kilodalton); Scramble, scrambke siRNA.

(Rho/rtTA) and with the tetracycline response element driving VEGF transcription (TRE-VEGF). Induction of VEGF expression was achieved by an initial gavage of doxycycline followed by delivery of doxycycline in the drinking water. To determine the effect of VEGF-induced retinal neovascularization on retinal vascular tight junction organization, retinas in mice with doxycycline-inducible VEGF expression from photoreceptors were removed 48 hours after administration of doxycycline. Immunofluorescence staining of the retinal whole mounts revealed $\mathrm{Ki}-67-$ positive proliferative cells were present only in vessels in the deep capillary plexus. Furthermore, dramatically altered occludin organization was observed on the endothelium of vessels within the deep capillary plexus, whereas occludin organization remained intact in the endothelium of vessels located in the superficial capillary plexus (Figure 5A). The images were scored in a masked manner by three unbiased observers using a scale of 1 to 5 and a clear change in occludin organization could be quantified in the deep capillary plexus (Figure 5B). In addition, organization of ZO-1 in retinal vasculature was severely altered in the vasculature within the deep capillary plexus and also moderately altered in the superficial capillary plexus vessels (Figure 5, A and B).

Doxycycline-inducible VEGF expression caused dramatic neovascularization by 3 days and retinal detachment beginning by 4 days $^{23}$ (Figure 6A). Blotting for occludin pS490 revealed a clear increase at 24 and 48 hours (Figure 6, B and C). Because the $r d 8$ mutation was observed in some mice in the inducible VEGF strain, the mutation was bred out of the strain and the experiment was further verified in mice in the absence of $r d 8$ mutation with the same response observed (Figure 6D). Furthermore, occludin could be observed in the centrosomes of endothelial cells in the deep capillary plexus of retinal whole mounts (Figure 6E). In control retinas, capillary occludin was well organized at the cell border and $\gamma$-tubulin, marking centrosomes, was observed adjacent to the cell border and colocalized with occludin. After VEGF induction with doxycycline, occludin border staining was lost but the colocalization of occludin with centrosomes was still clearly observed in proliferative endothelial cells. Furthermore, after VEGF induction pS490 occludin increased as puncta distributed in the cell cytoplasm, some of which clearly colocalize with centrosomes (Figure 6F). Collectively, these studies demonstrate that occludin phosphorylation on S490 is associated with VEGF-induced retinal neovascularization and at least some of occludin localizes to centrosomes in proliferating endothelial cells in vivo.

To further characterize loss of the BRB after induction of VEGF expression, the deposition in the retina of a crosslinkable, blood-borne marker was measured. Sulfo-NHSbiotin was delivered by transcardiac perfusion followed by flushing the vessels and fixing with paraformaldehyde. Retinas were removed for whole mount staining for covalently linked biotin adducts. In control animals, limited Sulfo-NHS-biotin staining could be observed and appeared restricted to the superficial capillary plexus (Figure 7A). After VEGF induction, extensive Sulfo-NHS-biotin staining was observed in the deep capillary plexus indicating increased vessel permeability along with Ki-67 staining (Figure 7, A and B). BRB permeability was also characterized by i.v. injection of fluorescein isothiocyanate-bovine serum albumin (fluorescein isothiocyanate-bovine serum albumin) followed by digital image analysis of retinal 
A Superficial plexus
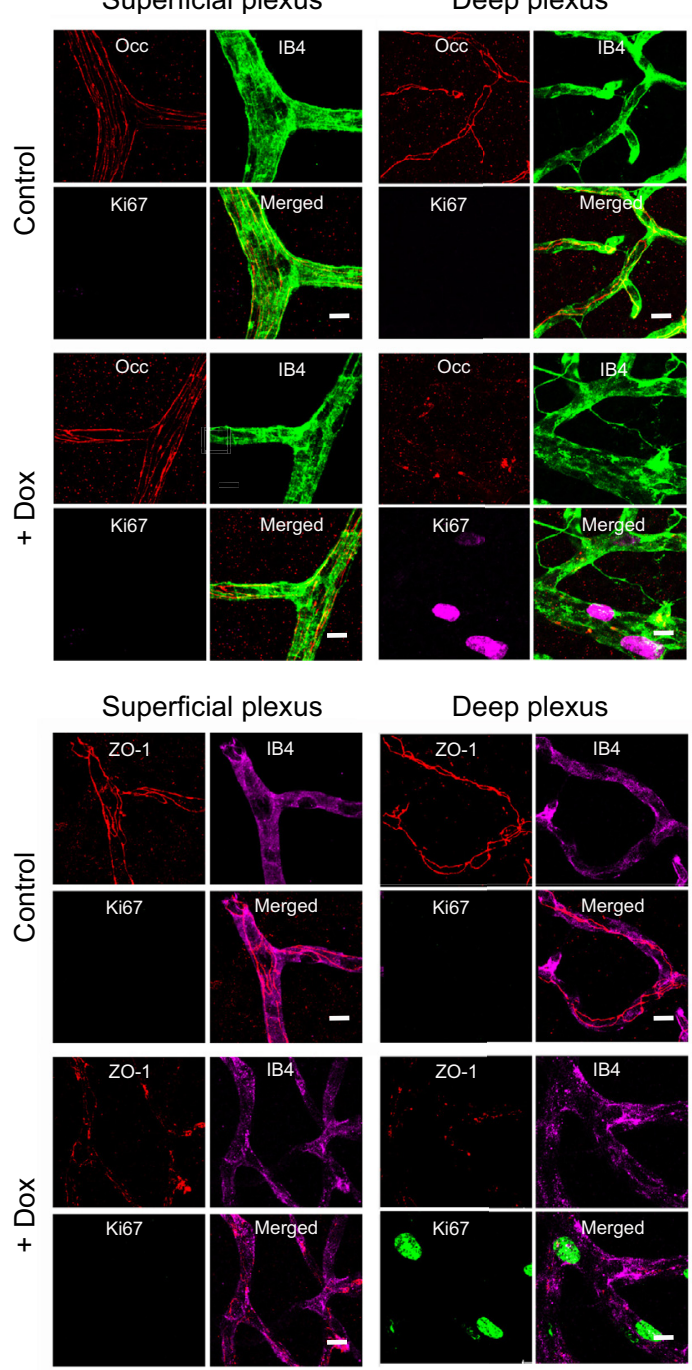

B
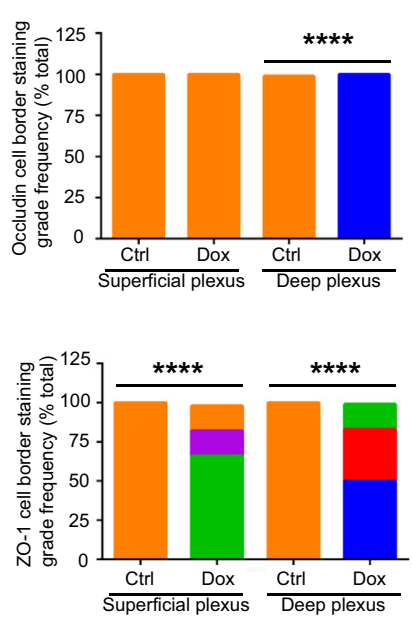

Grade 5 ( $0 \%$ loss) Grade 4 (1 25\% loss) Grade 3 (26 50\% loss) Grade 2 (51 75\% loss) Grade 1 (76 100\% loss)
Figure 5 The distribution of occludin and zonula occludens 1 (Z0-1) was altered in the deep capillary plexus during vascular endothelial growth factor (VEGF)-induced angiogenesis in vivo. A: Mouse retinal whole mounts obtained from Rho/rtTA(+) TRE-VEGF(+) mice after delivery of water (control) or doxcycline (Dox) for 48 hours were analyzed by immunohistochemistry (IHC) for occludin. Top panels: IHC for occludin (red), IB4 (green), and Ki-67 (purple) in both superficial plexus and deep plexus of retinal whole mounts. Bottom panels: IHC for ZO-1 (red), IB4 (purple), and Ki-67 (green). B: Staining of occludin or Z0-1 at the tight junction border was quantified by a rank scoring system based on a scale of 1 (near complete loss) to 5 (no loss) described in Materials and Methods. For each condition, images are from four random fields from three independent experiments. Data represent means \pm SEM with analysis by $\chi^{2}$ (B). $n=6$ eyes (B). $\star \star \star * P<0.0001$. Scale bar $=10 \mu \mathrm{m}(\mathbf{A})$. sections. After VEGF induction, retinal fluorescence intensity was increased by $28 \%$ (IPL), $59 \%$ (INL), $31 \%$ (OPL), and $32 \%$ (ONL) (Figure 7, C and D). These studies suggest that the deep capillary plexus responds to the VEGF expressed in the rods leading to occludin and ZO- 1 cell border reorganization and TJ disruption, permeability, occludin S490 phosphorylation, including centrosomal localization and proliferation.

\section{AAV2-Mediated Overexpression of Occludin S490A Inhibits Retinal Neovascularization in Vivo}

To determine whether occludin phosphorylation was required for retinal neovascularization, S490A occludin was delivered to the vasculature by virus with endothelial cell restricted expression, in the Rho/rtTA; TRE-VEGF mouse strain. Wt occludin and S490A mutant occludin expression specifically targeted to vascular endothelial cells was achieved using recombinant AAV serotype 2 (AAV2) quadruple tyrosine to phenylalanine (quadYF) capsid mutant vectors containing cDNA under control of the vascular endothelial cadherin promoter. ${ }^{24}$ Control experiments were conducted to demonstrate effective viral delivery and expression in endothelial cell culture and in the retina. Western blot analysis revealed that viral-mediated transduction of BRECs with Wt occludin, or S490A mutant resulted in 2.5- to 3-fold increase in occludin expression compared with GFP control, 4 days after transduction at a multiplicity of infection of 10,000 (Supplemental Figure S4, A and B). This observation was further confirmed by immunofluorescence staining with occludin mAb (Supplemental Figure S4C). Overexpression of occludin S490A caused approximately $70 \%$ reduction in VEGF-induced tube formation compared with GFP control and Wt Occludin (Supplemental Figure S4, D and E), and this result is consistent with the observation in BRECs transfected with plasmids containing GFP, Wt occludin, or S490A mutant occludin (Figure 3). Subretinal injection of AAV2 quadYF-GFP resulted in elevated expression of GFP by 3 weeks, specifically within the retinal vasculature as indicated by immunostaining of retina whole mounts and cross 


\section{A}

Rho/rtTA (+), TRE-VEGF (-) Rho/rtTA (+), TRE-VEGF (+)

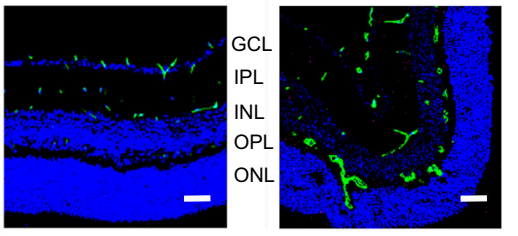

C

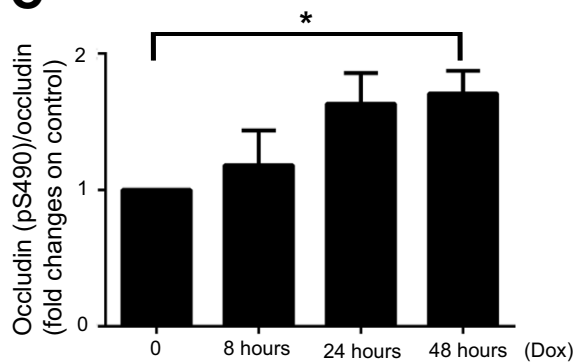

B

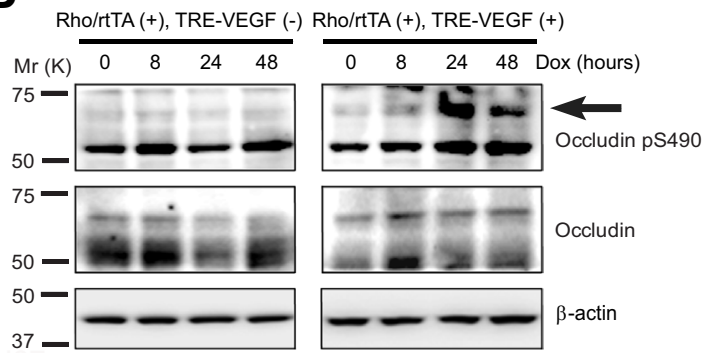

D

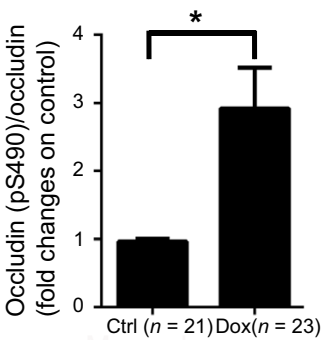

E
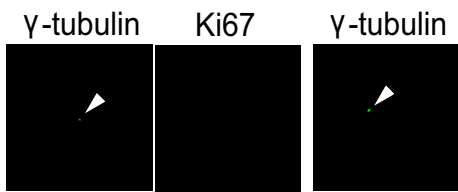

Ki67
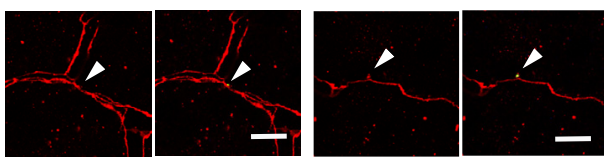

Occ Merged

Occ

Merged

Control
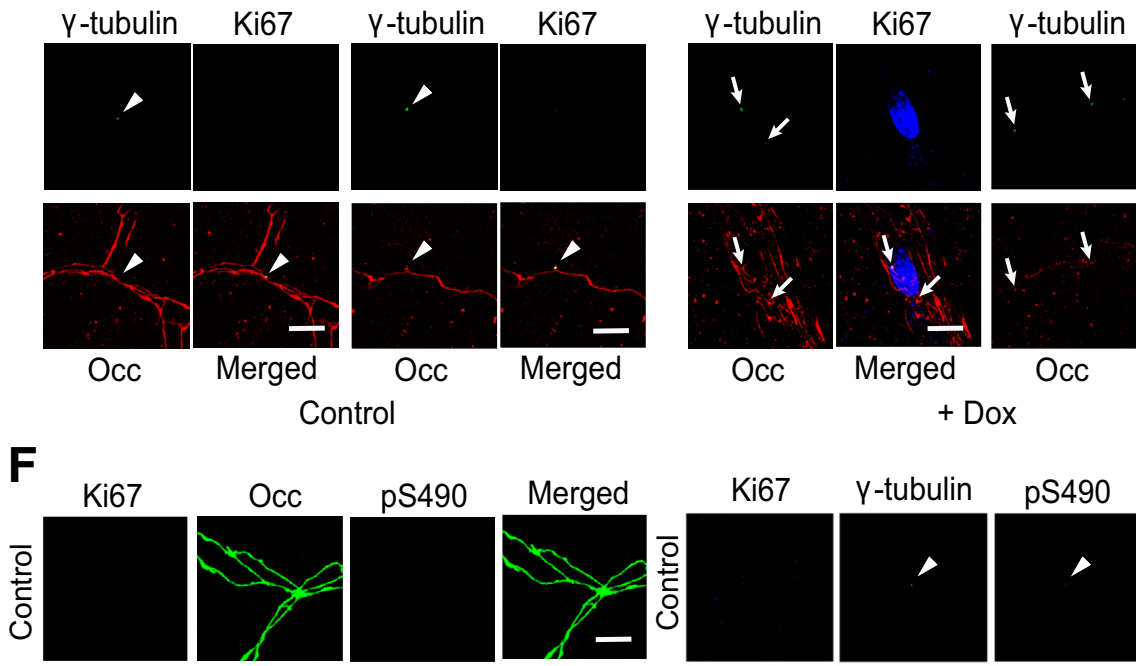

Ki67
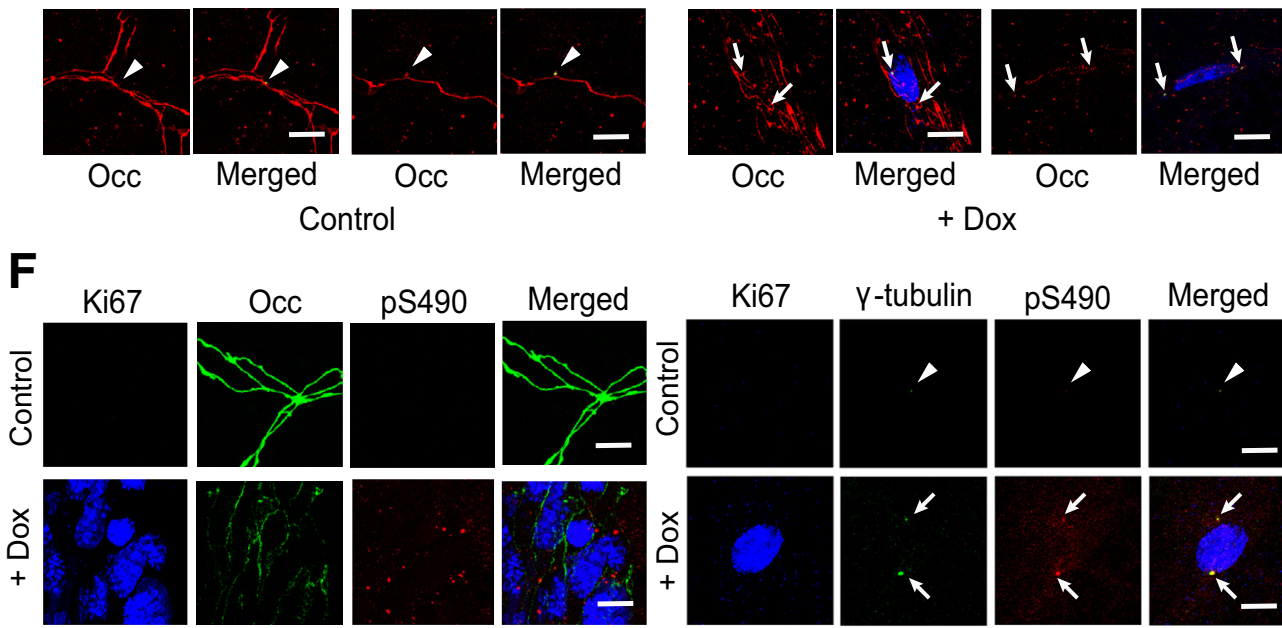

Occ

Merged

Occ

+ Dox

Figure 6 0ccludin S490 is phosphorylated during retinal angiogenesis. At postnatal day 21, vascular endothelial growth factor (VEGF) was induced from rods by delivery of doxycycline to rho/rtTA-TRE/VEGF double-transgenic mice. A: Visualization of blood vessels by IB4 (green) staining three days after VEGF induction, nucleus stained by DAPI (blue). Western blot analysis of occludin pS490 (B) and quantification (indicated by the arrow) (C) from three independent experiments. Data represent analysis by one-way analysis of variance with Bonferroni post-test. D: Quantification of pS490 at 48 hours after VEGF induction. Data represent analysis by Student's $t$-test. Mouse retinal whole mounts 48 hours after VEGF induction were analyzed by immunohistochemistry for $\gamma$-tubulin (green), polyclonal $\alpha$-occludin (red), Ki-67 (blue) (E), and Ki-67 (blue), monoclonal $\alpha$-occludin (green) or $\gamma$-tubulin (green), and occludin pS490 (red) (F). The arrows indicate the centrosomes of the proliferating cell, and the arrowheads indicate the centrosomes of the non-proliferating cell. Data represent means \pm SEM (C and $\mathbf{D})$. ${ }^{*} P<0.05$. Scale bars: 50 um (A); $10 \mu \mathrm{m}$ (E and $\left.\mathbf{F}\right)$.

sections (Supplemental Figure S5). These results clearly indicated the efficiency of AAV2 delivery to the vasculature through subretinal injection.

Retinal vascular endothelium of Rho/rtTA; TRE-VEGF mice were transduced with Wt Occ or S490A Occ at 3 weeks before induction of VEGF with doxycycline. Measures of angiogenesis by immunofluorescence staining using Isolectin B4 to identify and quantify blood vessels in retinal cross sections revealed that the increased angiogenesis induced by VEGF expression was blocked by viral delivery 

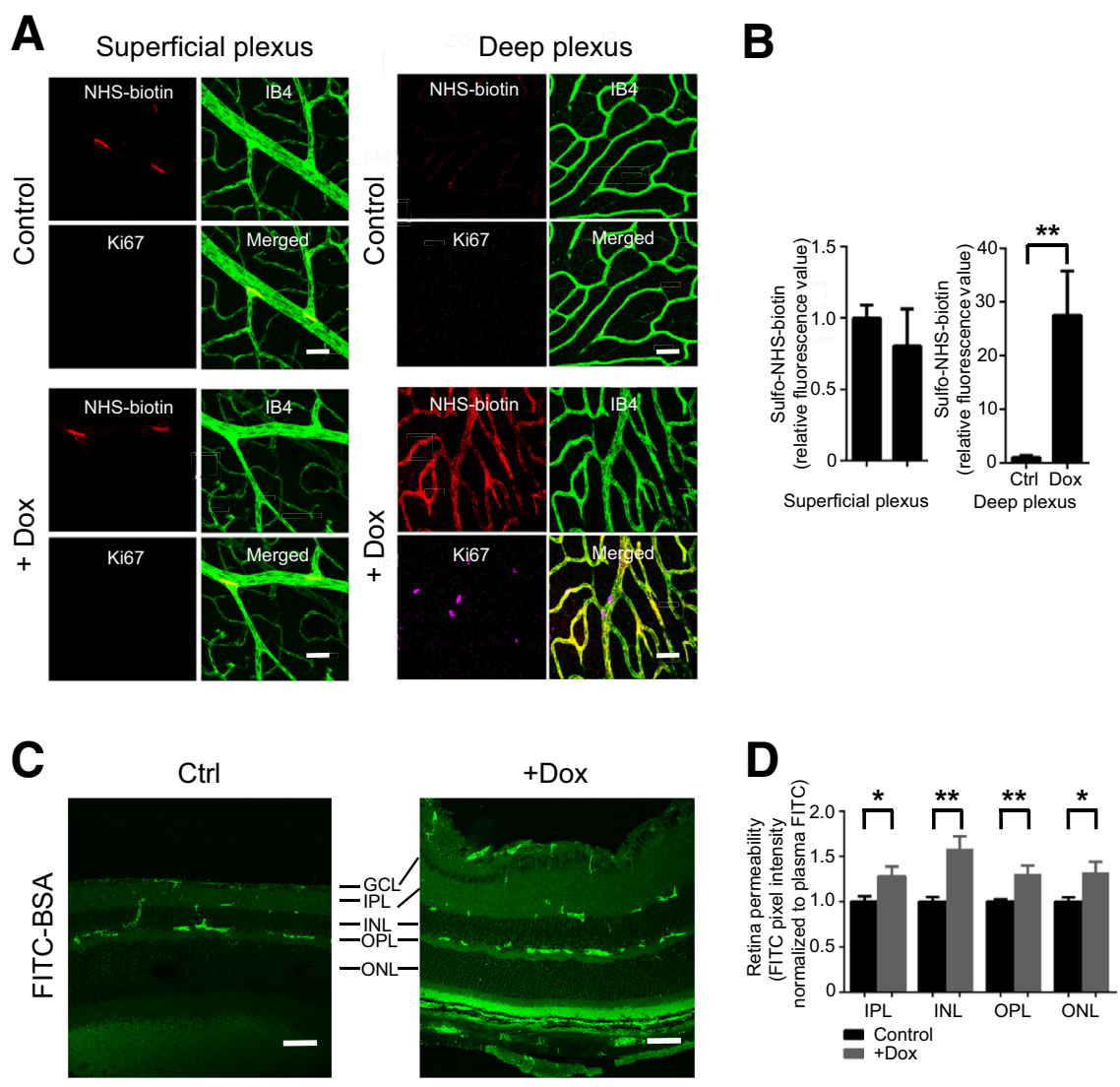

Figure 7 The integrity of blood-retinal barrier (BRB) is altered during vascular endothelial growth factor (VEGF)-induced angiogenesis in vivo. A: Mouse retinal whole mounts obtained from Rho/rtTA(+) TRE-VEGF(+) mice without doxycycline (Dox; control) or mice after VEGF induction with Dox that received intracardiac perfusion with sulfo-NHS-biotin to assess regions of permeability. BRB defect is detected more severely in deep capillary bed as compared to superficial plexus in inducible VEGF mice. B: SulfoNHS-biotin staining was quantified from images of four random fields from six eyes. C: Retinal cryosections from Rho/rtTA(+) TRE/VEGF(+) mice 48 hours after VEGF induction that received i.v. injection of fluorescein isothiocyanate (FITC)bovine serum albumin (green) stained with Hoechst (blue) to assess regions of permeability. D: FITC pixel intensity was quantified from images of four fields next to optic nerve region in serial sections from six eyes. Data represent means \pm SEM with analysis by Student's $t$-test as indicated in the graph (B and $\mathbf{D}) .{ }^{*} P<0.05,{ }^{*} P<0.01$. Scale bar $=50 \mu \mathrm{m}(\mathbf{A}$ and $\mathbf{C})$.

of S490A Occ compared to either Wt Occ or virus expressing GFP alone (Figure 8). In addition, the number of vessels in the outer retina was quantified because, normally there are no vessels in the outer nuclear layer but may grow there in response to VEGF expression induced from rods. In AAV2-GFP transduced animals with no doxycycline treatment, no vessels in the outer retina were observed (22 images in three animals) as expected. Doxycycline induction of VEGF in GFP-transduced animals led to 48 vessels in the outer retina in 115 images of 15 animals. WT Occ expression had no effect on this neovascularization with 42 vessels in the outer retina in 96 images of 13 animals. Expression of S490A Occ reversed the VEGF effect on neovascularization with three vessels in the outer retina in 118 images of 15 animals. These data provide compelling evidence that occludin phosphorylation on S490 is required for VEGF-induced retinal neovascularization in vivo.

\section{Discussion}

We report a novel function of occludin whereby phosphorylation on S490 is required for VEGF-induced neovascularization in retinal endothelial cells expressing tight junctions. Previous in vitro and in vivo studies have demonstrated that VEGF induces occludin phosphorylation within 15 to 30 minutes and is required for increased permeability of confluent endothelial monolayers. ${ }^{15,16}$
Herein, we demonstrate an associated but distinct phenomenon that subconfluent primary retinal endothelial cells in collagen matrices and retinal vascular endothelium respond to VEGF with increased S490 phosphorylation at centrosomes at 12 and 24 hours coincident with tube formation or neovascularization, respectively. Furthermore, phosphorylation of occludin on S490 localizes to centrosomes in neovascular epiretinal membranes from PDR patients, indicating this process is relevant to human disease. More important, transfection with an occludin S490A point mutant inhibited tube formation, proliferation, and migration in primary endothelial cells after VEGF treatment. Finally, transduction of occludin S490A mutant in endothelial cells by subretinal delivery, using a viral expression system, blocked VEGF-induced retinal neovascularization in vivo. Collectively, these studies reveal occludin is inhibitory to VEGF-induced neovascularization, which may be relieved by occludin knockdown or physiologically by occludin S490 phosphorylation.

The data suggest occludin S490 phosphorylation is required for angiogenesis but the $\mathrm{S} 490 \mathrm{D}$ phosphomimic was not sufficient to induce tube formation, which is not surprising given the broad array of cell cycle control points regulated by receptor tyrosine kinases. Previous studies demonstrate that other junctional proteins also contribute to both barrier properties and angiogenesis. The tight junction proteins ZO-1 and ZO-2 have been shown to contribute to growth control in a number of reports. ${ }^{9,30}$ Gene deletion of 
A

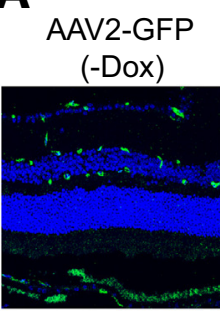

AAV2-WT Occ AAV2-S490A Occ (+Dox)

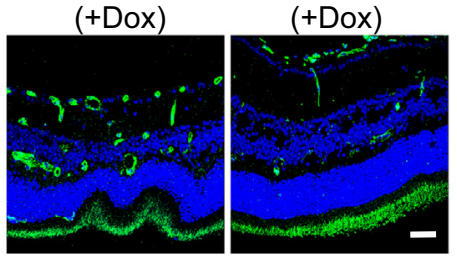

B

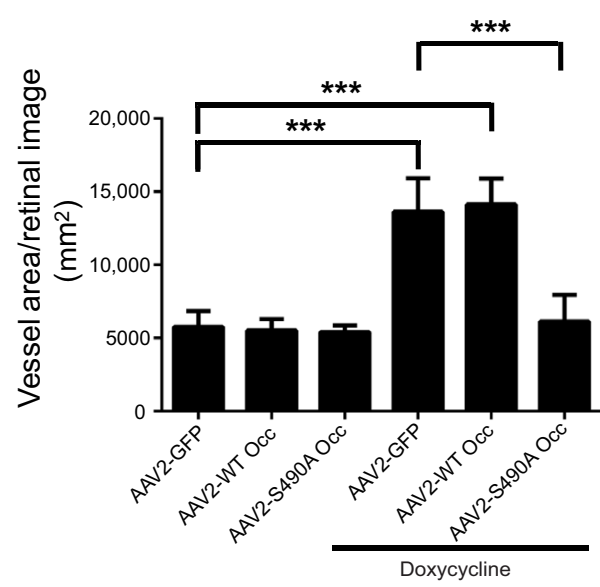

Figure 8 Occludin $S 490 \mathrm{~A}$ inhibits angiogenesis in vivo. AAV2-GFP, AAV2-Wt Occ, or AAV2S490A Occ was delivered to Rho/rtTA(+) TRE$\operatorname{VEGF}(+)$ mice through subretinal injection. After 3 weeks, vascular endothelial growth factor (VEGF) in mouse retinas was induced by administration of doxycycline (Dox) for 3 days. Retinas were isolated and prepared for imaging. A: Immunohistochemical staining of IB4 (green) and DAPI (blue) on retinal cryostat sections. B: Vessel area was quantified with an $n=3,3,3,12,10$, and 12 , respectively. Data represent means \pm SEM with analysis by one-way analysis of variance with Bonferroni post-test (B). ${ }^{* * * P}<0.001$. Scale bar $=50 \mu \mathrm{m}(\mathbf{A})$.
ZO-1 leads to embryonic lethality with disturbed yolk sac angiogenesis and delayed embryonic growth from embryonic day (E)8.5 onwards with significant notochord apoptosis. ${ }^{31}$ ZO-2 gene deletion is also embryonic lethal shortly after implantation because of an arrest in early gastrulation and disturbed mesodermal differentiation also with decreased proliferation and increased apoptosis. ${ }^{32}$ Tornavaca et $\mathrm{al}^{33}$ have recently demonstrated that $\mathrm{ZO}-1$ contributes to control of angiogenesis with a reduction in tube formation in cell culture assays and reduced angiogenesis in matrigel plug assay in response to FGF after ZO1 knockdown. These studies demonstrate a requirement for the tight junction proteins ZO-1 and ZO-2 in growth control and survival.

A role for the adherens junction protein $\beta$-catenin in growth control in a number of cell types is well established, and in vascular endothelium VE-cadherin and $\beta$-catenin contribute to the inhibition of VEGF-induced endothelial proliferation by cell contact. ${ }^{34}$ In addition, Wnt signaling through $\beta$-catenin stabilization is required for claudin 3 transcriptional control and barrier induction, ${ }^{35}$ whereas in adults, Wnt signaling through $\beta$-catenin can promote vascular permeability. ${ }^{36}$ Conditional gene deletion of $\beta$ catenin in endothelial cells leads to embryonic lethality at E11.5 to 13.5 with defects in specific vascular beds, including head, large vitelline and umbilical vessels, and placenta. Blood vessels demonstrated increased hemorrhage, less organized vascular networks, smaller or irregular diameter, and dead-ending vessels. ${ }^{37}$ More important, angiogenesis in the central nervous system is specifically inhibited with virtually no capillaries in the forebrain and spinal cord. ${ }^{38}$ Therefore, the junctional protein $\beta$-catenin contributes to angiogenesis specifically in tissues with a well-developed junctional complex like the blood-brain barrier.

The role of occludin S490 phosphorylation in neovascularization likely relates to its centrosomal localization. Consistent with previous findings in epithelial cells, ${ }^{17}$ the current study reveals that occludin localizes to centrosomes with an increase in S490 phosphorylation during mitosis. S490 phosphorylated occludin localizes to centrosomes of proliferating endothelial cells, retinal vasculature of mice with VEGF-induced angiogenesis, and epiretinal membranes from human PDR patients. In epithelial cells, the S490 phosphorylation was shown to regulate centrosome separation and mitotic entry ${ }^{17}$ and here S490A expression completely prevented VEGF-induced tube formation or proliferation. Centrosomes contribute to the maintenance of cell polarity during migration, tissue growth, and homeostasis and are critical for signal transduction. ${ }^{39,40}$ The centriole linker complex that bridges the mother and daughter centrosomes contains the adherens junction protein $\beta$-catenin, which participates in the reorganization of the linker and centrosome separation in a NEK2 kinase dependent manner. $^{41,42}$ Further characterization of how occludin may interact with these centrosome proteins may provide more detailed molecular understanding of the role of occludin in regulation of angiogenesis.

Occludin may contribute to angiogenesis in addition to a centrosomal function. Because alterations in cell adhesion may alter cell migration in the angiogenic tube, ${ }^{43}$ the changes in occludin phosphorylation may contribute to altered cell migration. Furthermore, occludin also regulates directional migration of epithelial cells through organization of the atypical protein kinase C, Par3, PATJ polarity complex at the cell leading edge, as demonstrated by gene knockdown experiments. ${ }^{44}$ These studies demonstrate a role for occludin Tyr 474 (human) phosphorylation in PI 3-kinase activation and cell migration. The S490A mutation inhibited both proliferation as measured by DNA synthesis and migration toward VEGF.

Transgenic mice with doxycycline inducible VEGF expression in photoreceptors develop neovascularization that originates from the deep capillary bed of the retina and causes outer retinal folds followed by total retinal detachment in 3 to 4 days after administration of doxycycline. ${ }^{23}$ This mouse model allowed investigation of occludin S490 
phosphorylation from vasculature with preformed tight junctions. Two days after VEGF induction, organization of tight junction proteins occludin and ZO-1 was dramatically altered in the deep capillary bed. Occludin organization was altered more severely than ZO-1 in the deep capillary bed but remained intact in superficial vessels where ZO-1 organization was also partially altered. Furthermore, we observed loss of BRB integrity, increased permeability, specifically in the deep capillary bed in association with loss of occludin border staining, increased occludin phosphorylation, and phospho-occludin staining at centrosomes. More important, neovascularization could be blocked by viral delivery of S490A occludin. These studies represent a pathological neovascularization, and it will be important to determine whether occludin contributes to vessel development and physiological angiogenesis.

These studies demonstrate an essential role for the tight junction protein occludin in pathological retinal angiogenesis induced by VEGF. The data suggest that occludin contributes an important control point for VEGF-driven permeability and angiogenesis. Blocking occludin phosphorylation at S490 or delivery of the dominant negative S490A mutant may provide a potential therapeutic approach for treatment of diseases with pathological neovascularization that target the endothelial cells without adverse effects on the surrounding parenchyma.

\section{Acknowledgments}

We thank Dr. Stephen Lentz for technical assistance on confocal microscopy; Dr. Cheng-mao Lin and Heather Lindner for preparing experimental animals; Dr. Jingyu Yao and Lin Jia for technical assistance on subretinal injections; Madeline Merlino for digital image analysis; and Dr. Peter Campochiaro for use of the rho/rtTA-TRE/VEGF mice.

X.L., A.D., M.D.-C., and E.A.R. performed the experiments; V.A.C. and W.W.H. provided reagents (AAV vectors); T.W.G. provided clinical samples; X.L., A.D., M.D.-C., E.A.R., T.W.G., and D.A.A. analyzed the data; and X.L. and D.A.A. designed the research and wrote the manuscript.

\section{Supplemental Data}

Supplemental material for this article can be found at http://dx.doi.org/10.1016/j.ajpath.2016.04.018.

\section{References}

1. Antonetti DA, Klein R, Gardner TW: Diabetic retinopathy. N Engl J Med 2012, 366:1227-1239

2. Titchenell PM, Antonetti DA: Using the past to inform the future: antiVEGF therapy as a road map to develop novel therapies for diabetic retinopathy. Diabetes 2013, 62:1808-1815

3. Miller JW, Adamis AP, Aiello LP: Vascular endothelial growth factor in ocular neovascularization and proliferative diabetic retinopathy. Diabetes Metab Rev 1997, 13:37-50
4. Nguyen QD, Brown DM, Marcus DM, Boyer DS, Patel S, Feiner L, Gibson A, Sy J, Rundle AC, Hopkins JJ, Rubio RG, Ehrlich JS: Ranibizumab for diabetic macular edema: results from 2 phase III randomized trials: RISE and RIDE. Ophthalmology 2012, 119:789-801

5. Do DV, Nguyen QD, Boyer D, Schmidt-Erfurth U, Brown DM, Vitti R, Berliner AJ, Gao B, Zeitz O, Ruckert R, Schmelter T, Sandbrink R, Heier JS; da Vinci Study Group: One-year outcomes of the da Vinci Study of VEGF Trap-Eye in eyes with diabetic macular edema. Ophthalmology 2012, 119:1658-1665

6. Lanzetta P, Mitchell P, Wolf S, Veritti D: Different antivascular endothelial growth factor treatments and regimens and their outcomes in neovascular age-related macular degeneration: a literature review. $\mathrm{Br}$ J Ophthalmol 2013, 97:1497-1507

7. Saitou M, Furuse M, Sasaki H, Schulzke JD, Fromm M, Takano H, Noda T, Tsukita S: Complex phenotype of mice lacking occludin, a component of tight junction strands. Mol Biol Cell 2000, 11: $4131-4142$

8. Schulzke JD, Gitter AH, Mankertz J, Spiegel S, Seidler U, Amasheh S, Saitou M, Tsukita S, Fromm M: Epithelial transport and barrier function in occludin-deficient mice. Biochim Biophys Acta 2005, 1669:34-42

9. Runkle EA, Mu D: Tight junction proteins: from barrier to tumorigenesis. Cancer Lett 2013, 337:41-48

10. Phillips BE, Cancel LM, Tarbell JM, Antonetti DA: Occludin regulates permeability under hydrostatic pressure and cell division in retinal pigment epithelial cells. Invest Ophthalmol Vis Sci 2008, 49: $2568-2576$

11. Wang Z, Mandell KJ, Parkos CA, Mrsny RJ, Nusrat A: The second loop of occludin is required for suppression of Raf1-induced tumor growth. Oncogene 2005, 24:4412-4420

12. Barrios-Rodiles M, Brown KR, Ozdamar B, Bose R, Liu Z, Donovan RS, Shinjo F, Liu Y, Dembowy J, Taylor IW, Luga V, Przulj N, Robinson M, Suzuki H, Hayashizaki Y, Jurisica I, Wrana JL: High-throughput mapping of a dynamic signaling network in mammalian cells. Science 2005, 307:1621-1625

13. Cummins PM: Occludin: one protein, many forms. Mol Cell Biol 2012, 32:242-250

14. Sundstrom JM, Tash BR, Murakami T, Flanagan JM, Bewley MC, Stanley BA, Gonsar KB, Antonetti DA: Identification and analysis of occludin phosphosites: a combined mass spectrometry and bioinformatics approach. J Proteome Res 2009, 8:808-817

15. Murakami T, Felinski EA, Antonetti DA: Occludin phosphorylation and ubiquitination regulate tight junction trafficking and vascular endothelial growth factor-induced permeability. J Biol Chem 2009, 284:21036-21046

16. Murakami T, Frey T, Lin C, Antonetti DA: Protein kinase cbeta phosphorylates occludin regulating tight junction trafficking in vascular endothelial growth factor-induced permeability in vivo. Diabetes 2012, 61:1573-1583

17. Runkle EA, Sundstrom JM, Runkle KB, Liu X, Antonetti DA: Occludin localizes to centrosomes and modifies mitotic entry. J Biol Chem 2011, 286:30847-30858

18. Tavana H, Jovic A, Mosadegh B, Lee QY, Liu X, Luker KE, Luker GD, Weiss SJ, Takayama S: Nanolitre liquid patterning in aqueous environments for spatially defined reagent delivery to mammalian cells. Nat Mat 2009, 8:736-741

19. Aijaz S, D'Atri F, Citi S, Balda MS, Matter K: Binding of GEF$\mathrm{H} 1$ to the tight junction-associated adaptor cingulin results in inhibition of Rho signaling and G1/S phase transition. Dev Cell 2005, 8:777-786

20. Harhaj NS, Felinski EA, Wolpert EB, Sundstrom JM, Gardner TW, Antonetti DA: VEGF activation of protein kinase $\mathrm{C}$ stimulates occludin phosphorylation and contributes to endothelial permeability. Invest Ophthalmol Vis Sci 2006, 47:5106-5115

21. Harhaj NS, Barber AJ, Antonetti DA: Platelet-derived growth factor mediates tight junction redistribution and increases permeability in MDCK cells. J Cell Physiol 2002, 193:349-364 
22. Koyama S, Takagi H, Otani A, Suzuma K, Nishimura K, Honda Y: Tranilast inhibits protein kinase C-dependent signalling pathway linked to angiogenic activities and gene expression of retinal microcapillary endothelial cells. Br J Pharmacol 1999, 127:537-545

23. Ohno-Matsui K, Hirose A, Yamamoto S, Saikia J, Okamoto N, Gehlbach P, Duh EJ, Hackett S, Chang M, Bok D, Zack DJ, Campochiaro PA: Inducible expression of vascular endothelial growth factor in adult mice causes severe proliferative retinopathy and retinal detachment. Am J Pathol 2002, 160:711-719

24. Qi X, Cai J, Ruan Q, Liu L, Boye SL, Chen Z, Hauswirth WW, Ryals RC, Shaw L, Caballero S, Grant MB, Boulton ME: GammaSecretase inhibition of murine choroidal neovascularization is associated with reduction of superoxide and proinflammatory cytokines. Invest Ophthalmol Vis Sci 2012, 53:574-585

25. Yao J, Feathers KL, Khanna H, Thompson D, Tsilfidis C, Hauswirth WW, Heckenlively JR, Swaroop A, Zacks DN: XIAP therapy increases survival of transplanted rod precursors in a degenerating host retina. Invest Ophthalmol Vis Sci 2011, 52:1567-1572

26. Yao J, Jia L, Khan N, Zheng QD, Moncrief A, Hauswirth WW, Thompson DA, Zacks DN: Caspase inhibition with XIAP as an adjunct to AAV vector gene-replacement therapy: improving efficacy and prolonging the treatment window. PLoS One 2012, 7:e37197

27. Antonetti DA, Barber AJ, Khin S, Lieth E, Tarbell JM, Gardner TW; Penn State Retina Research Group: Vascular permeability in experimental diabetes is associated with reduced endothelial occludin content: vascular endothelial growth factor decreases occludin in retinal endothelial cells. Diabetes 1998, 47:1953-1959

28. Muthusamy A, Lin CM, Shanmugam S, Lindner HM, Abcouwer SF, Antonetti DA: Ischemia-reperfusion injury induces occludin phosphorylation/ubiquitination and retinal vascular permeability in a VEGFR-2dependent manner. J Cereb Blood Flow Metab 2014, 34:522-531

29. Aiello LP, Avery RL, Arrigg PG, Keyt BA, Jampel HD, Shah ST, Pasquale LR, Thieme H, Iwamoto MA, Park JE, Nguyen HV, Aiello LM, Ferrara N, King GL: Vascular endothelial growth factor in ocular fluid of patients with diabetic retinopathy and other retinal disorders. N Engl J Med 1994, 331:1480-1487

30. Bauer H, Zweimueller-Mayer J, Steinbacher P, Lametschwandtner A, Bauer HC: The dual role of zonula occludens (ZO) proteins. J Biomed Biotechnol 2010, 2010:402593

31. Katsuno T, Umeda K, Matsui T, Hata M, Tamura A, Itoh M, Takeuchi K, Fujimori T, Nabeshima Y, Noda T, Tsukita S, Tsukita S: Deficiency of zonula occludens-1 causes embryonic lethal phenotype associated with defected yolk sac angiogenesis and apoptosis of embryonic cells. Mol Biol Cell 2008, 19:2465-2475

32. Xu J, Kausalya PJ, Phua DC, Ali SM, Hossain Z, Hunziker W: Early embryonic lethality of mice lacking ZO-2, but not ZO-3, reveals critical and nonredundant roles for individual zonula occludens proteins in mammalian development. Mol Cell Biol 2008, 28:1669-1678
33. Tornavaca O, Chia M, Dufton N, Almagro LO, Conway DE, Randi AM, Schwartz MA, Matter K, Balda MS: ZO-1 controls endothelial adherens junctions, cell-cell tension, angiogenesis, and barrier formation. J Cell Biol 2015, 208:821-838

34. Grazia Lampugnani M, Zanetti A, Corada M, Takahashi T, Balconi G, Breviario F, Orsenigo F, Cattelino A, Kemler R, Daniel TO, Dejana E: Contact inhibition of VEGF-induced proliferation requires vascular endothelial cadherin, beta-catenin, and the phosphatase DEP-1/CD148. J Cell Biol 2003, 161:793-804

35. Liebner S, Corada M, Bangsow T, Babbage J, Taddei A, Czupalla CJ, Reis M, Felici A, Wolburg H, Fruttiger M, Taketo MM, von Melchner H, Plate KH, Gerhardt H, Dejana E: Wnt/beta-catenin signaling controls development of the blood-brain barrier. J Cell Biol 2008, 183:409-417

36. Bai Y, Ma JX, Guo J, Wang J, Zhu M, Chen Y, Le YZ: Muller cellderived VEGF is a significant contributor to retinal neovascularization. J Pathol 2009, 219:446-454

37. Cattelino A, Liebner S, Gallini R, Zanetti A, Balconi G, Corsi A, Bianco P, Wolburg H, Moore R, Oreda B, Kemler R, Dejana E: The conditional inactivation of the beta-catenin gene in endothelial cells causes a defective vascular pattern and increased vascular fragility. J Cell Biol 2003, 162:1111-1122

38. Daneman R, Agalliu D, Zhou L, Kuhnert F, Kuo CJ, Barres BA: Wnt/beta-catenin signaling is required for CNS, but not non-CNS, angiogenesis. Proc Natl Acad Sci U S A 2009, 106:641-646

39. Bornens M: The centrosome in cells and organisms. Science 2012 , 335:422-426

40. Dormoy V, Tormanen K, Sutterlin C: Par6gamma is at the mother centriole and controls centrosomal protein composition through a Par6alpha-dependent pathway. J Cell Sci 2013, 126:860-870

41. Bahmanyar S, Kaplan DD, Deluca JG, Giddings TH Jr, O'Toole ET, Winey M, Salmon ED, Casey PJ, Nelson WJ, Barth AI: Beta-Catenin is a Nek2 substrate involved in centrosome separation. Genes Dev 2008, 22:91-105

42. Zheng XY, Wu YT, Zhu LF, Chen QS, Zhou YJ, Yan H, Chen T, Xiao QZ, Zhu JH, Zhang L: Angiotensin II promotes differentiation of mouse embryonic stem cells to smooth muscle cells through PI3kinase signaling pathway and NF-kappa B. Differentiation 2013, 85: $41-54$

43. Bentley K, Franco CA, Philippides A, Blanco R, Dierkes M, Gebala V, Stanchi F, Jones M, Aspalter IM, Cagna G, Westrom S, ClaessonWelsh L, Vestweber D, Gerhardt H: The role of differential VEcadherin dynamics in cell rearrangement during angiogenesis. Nat Cell Biol 2014, 16:309-321

44. Du D, Xu F, Yu L, Zhang C, Lu X, Yuan H, Huang Q, Zhang F, Bao H, Jia L, Wu X, Zhu X, Zhang X, Zhang Z, Chen Z: The tight junction protein, occludin, regulates the directional migration of epithelial cells. Dev Cell 2010, 18:52-63 